\title{
Interação em Interfaces de Softwares de Geometria Interativa: Um Mapeamento Sistemático
}

\author{
Helena M. Reis ${ }^{1}$, Simone S. Borges ${ }^{1}$, Paul A. Griffiths, Luis F. S. Moro ${ }^{1}$, \\ Seiji Isotani ${ }^{1}$ \\ ${ }^{1}$ Universidade de São Paulo - Instituto de Ciências Matemáticas e Computação (ICMC) \\ Caixa Postal 668 - CEP 13560-970 - São Carlos - SP - Brazil \\ \{helenamcd, sborges, luismoro, sisotani\}@icmc.usp.br, \\ truegriffin@gmail.com
}

\begin{abstract}
The interaction mode via Interactive Geometry interfaces can influence geometry learning. Nevertheless, approaches to the development of these interfaces are poorly investigated. To provide an overview of the results already obtained in this area, a systematic mapping was conducted in order to analyze which types of interfaces (i.e. forms of interaction) have been developed and in which devices these interfaces have been used. In total, 998 studies were analyzed in more than 10 years of research in the area of interactive geometry. Among these, 45 studies were related to the development of interfaces and only 20 of them satisfied the inclusion and exclusion criteria defined in this work. As a result, we verified that most of the studies have used created interfaces for desktops based on keyboard and mouse. We also identified that there are several opportunities to research new types of user interfaces and interactions to support geometry learning.
\end{abstract}

Resumo. $O$ modo de interação por meio das interfaces dos softwares de Geometria Interativa pode influenciar no aprendizado da geometria. Apesar disto, as abordagens sobre o desenvolvimento destas interfaces são pouco investigadas. Para oferecer uma visão geral dos resultados já obtidos nesta área, um mapeamento sistemático foi conduzido com o objetivo de analisar quais tipos de interfaces (i.e. formas de interação) vem sendo desenvolvidas e em quais dispositivos estas interfaces são utilizadas. No total foram 998 estudos analisados em mais de 10 anos de pesquisa na área de geometria interativa. Dentre estes estudos 45 deles estavam relacionados com o desenvolvimento de interfaces e apenas 20 deles satisfizeram os critérios de inclusão e exclusão definidos neste trabalho. Como resultado, verificou-se que maioria dos estudos apresentam interfaces baseadas nos computadores de mesa que possuem interação por meio do teclado e mouse e que existem diversas oportunidades de pesquisa na geração de novos tipos de interface e interação para aprendizagem de geometria.

\section{Introdução}

Os softwares de Geometria Interativa (GI) surgiram no início da década de 90 com o objetivo de possibilitar aos estudantes explorarem a geometria por meio do computador (Roanes-Lozano, 2003). Estes softwares permitem a aprendizagem da geometria por meio da manipulação dinâmica dos objetos geométricos, como, por exemplo, retas, 
circunferências e pontos (Isotani e Brandão 2004; Erez e Yerushalmy 2007; RoanesLozano 2003). É por meio da interface que os estudantes interagem com o software e podem visualizar suas construções geométricas, além de interagir com as funcionalidades disponíveis, o que pode facilitar o entendimento de informações mais complexas (Shimomura, Havannber e Hafsteinsson, 2013). Laborde (2007) comenta que os desenvolvedores dos softwares de GI deveriam se preocupar tanto com os aspectos pedagógicos quanto com o design da interface. Isto por que interfaces indevidamente projetadas e desenvolvidas podem causar frustrações nos alunos, fazendo com que eles sintam dificuldade em utilizar o software, terminando por não direcionar a sua atenção para aprendizado da geometria (Schimpf e Spannagel 2001; Kotenkamp e Dohrman 2010). Entretanto, a despeito desta importância, estudos sobre as várias formas de interação disponíveis para a criação de interfaces para softwares de GI não tem sido amplamente explorados. Existem poucos estudos na área que exploram esta diversidade de interações e os dispositivos em que são executados.

Dessa forma, este trabalho apresenta os resultados de um mapeamento sistemático elaborado com o propósito de investigar quais formas de interação vêm sendo mais utilizadas no estudo e desenvolvimento de interfaces para softwares de GI, e para quais dispositivos estes softwares foram desenvolvidos. Mapeamentos sistemáticos são uma forma de categorizar e sintetizar informações existentes sobre uma determinada área de interesse, respondendo a questões de pesquisa de modo imparcial (Petersen 2008). O restante deste trabalho está organizado da seguinte forma: na Seção 2 é fornecida a metodologia de pesquisa, a definição das questões de pesquisa e a condução das atividades de busca. A Seção 3 apresenta os resultados deste mapeamento e responde as questões de pesquisa. A Seção 4 apresenta a discussão dos resultados obtidos, Seção 5 discute eventuais ameaças à validade e, por fim, na Seção 6 é apresentada a discussão e conclusão.

\section{Método de pesquisa}

O objetivo deste artigo é apresentar os resultados do mapeamento sistemático elaborado para fornecer uma visão geral dos estudos existentes que apresentam interfaces de software para GI. O objetivo secundário é obter uma visão geral sobre para quais dispositivos este tipo de software tem sido mais desenvolvido. Este estudo baseou-se nas diretrizes propostas por Petersen et al. (2008) e foi conduzido de acordo com os cinco passos a seguir: (i) definição de questões de pesquisa, (ii) realização da pesquisa de estudos primários relevantes, (iii) triagem dos documentos, (iv) keywording dos resumos, e (v) extração de dados e mapeamento.

\subsection{Definição de questões de pesquisa}

As questões de pesquisas definidas neste estudo são as seguintes:

$\mathbf{Q P}_{1}$ : Quais são as principais formas ${ }^{1}$ de interação investigadas para o desenvolvimento de interfaces de softwares de GI?

$\mathbf{Q P}_{2}$ : Quais são os principais dispositivos para os quais softwares de GI tem sido desenvolvidos?

\footnotetext{
${ }^{1}$ Possíveis formas de entrada (e.g. mouse ou teclado) e saída (e.g. representação 2D ou 3D) de dados para construir objetivos geométricos
} 


\subsection{Condução da pesquisa}

As bibliotecas digitais utilizadas para se obter os estudos primários foram: IEEExplore, ACM Digital Library, Scopus, Elsevier (via Science Direct) e Springer Link (via Science Direct). Para realizar a busca desses estudos foi utilizada uma string de pesquisa na língua inglesa (ver Tabela 1). Os autores optaram por utilizar a string de pesquisa somente em língua portuguesa devido a preferencia por artigos indexados em base de dados consolidadas. A string é constituída de duas partes, e formatada de acordo com as regras de cada biblioteca digital. A primeira parte relaciona ao ensino da geometria por meio dos softwares de GI e a segunda parte abrange os estudos relacionados com as interfaces destes softwares de GI. Na Tabela 1 o operador boolean $O R$ conecta os os sinônimos da string de pesquisa, enquanto o operador $A N D$ conecta as partes. Nenhum campo específico (filtro) foi utilizado para limitar as buscas, como por exemplo, data de publicação.

Tabela 1 - String de pesquisa em inglês.

\begin{tabular}{l|l}
\hline Palavra-chave & Termos alternativos e/ou sinônimos \\
\hline Interactive geometry & "Dynamic Geometry" OR "Geometry Teaching" OR "Geometry Education” AND \\
Interface & $\begin{array}{l}\text { HCI OR "Human-Computer Interaction" OR "Man Computer Interaction” OR "Human } \\
\text { Factors" OR "User Interface" OR "Computer Interface" OR "Gestural Interface” OR } \\
\text { "Human Information Processing" OR gesture OR usability }\end{array}$ \\
\hline
\end{tabular}

Os estudos selecionados foram analisados a fim de que fossem identificados aqueles relevantes ao contexto da pesquisa. Esta análise consistiu na aplicação de critérios de inclusão e exclusão previamente definidos. Os critérios de inclusão elaborados foram os seguintes:

- Se vários artigos mostram estudos similares, somente o mais recente deverá ser incluído;

- Se houver várias versões do mesmo artigo, e.g. uma resumida e outra completa, a mais completa deverá ser incluída;

- Artigos que descrevem o desenvolvimento ou análise de interfaces de softwares de Geometria Interativa em qualquer plataforma.

Critérios de exclusão são importantes, pois permitem uma maior precisão na eliminação de estudos considerados não relevantes ao contexto da pesquisa em andamento. Por essa razão, durante a análise dos estudos retornados, todos aqueles que enquadraram-se em ao menos um dos critérios de exclusão abaixo foram descartados.

- Artigos que não apresentem relação com interfaces de softwares de Geometria Interativa serão excluídos;

- Estudos que não estejam na língua portuguesa ou inglesa serão excluídos;

- Relatórios técnicos, documentos que estão disponíveis na forma de resumos ou apresentações/slides e também estudos secundários (ou seja, revisões e mapeamentos sistemáticos da literatura) serão excluídos.

A pesquisa dos estudos primários foi realizada no período do dia 16/05/2013 ao dia 30/05/2013. Primeiramente foram retornados 998 estudos primários das bibliotecas digitais apresentadas na Tabela 2. Dois autores deste estudo realizaram simultaneamente 
a leitura dos títulos, resumos e palavras-chave, de todos os artigos e cada um gerou a lista de estudos selecionados. Os dois autores discutiram sobre quais estudos deveriam ser selecionados, e ao final o conjunto inicial de 998 foi reduzido à somente 45 estudos realmente relacionados ao tópico investigado. Após este passo, foi realizada novamente a leitura dos resumos, introdução e conclusão e a aplicação dos critérios de inclusão e exclusão, o que resultou em um subconjunto de 20 estudos. Em seguida foi realizada a leitura integral dos estudos selecionados a fim de se proceder a categorização dos mesmos. Esta etapa será discutida mais detalhadamente na próxima seção.

\section{Tabela 2 - Quantidade de estudos retornados por cada base de dados} eletrônica, total de estudos candidatos e seleção final.

\begin{tabular}{l|r}
\hline \multicolumn{1}{c|}{ Base de dados } & \multicolumn{1}{c}{ Quantidade } \\
\hline ACM Digital Library & 385 \\
Scopus & 338 \\
Elsevier (via Science Direct) & 243 \\
Springer Link (via Science Direct) & 29 \\
IEEExplore & 12 \\
\hline Total & $\mathbf{9 9 8}$ \\
Candidatos & $\mathbf{4 5}$ \\
Seleção final & $\mathbf{2 0}$ \\
\hline
\end{tabular}

Cada estudo primário foi categorizado de acordo com as formas de interação (entrada e saída de dados) com as interfaces dos softwares de GI encontrada nos trabalhos analisados. Cada estudo foi classificado em uma ou mais categorias identificadas abaixo, sendo como saída de dados as seguintes categorias:

3D: Nesta categoria foram incluídos todos os estudos que apresentam softwares de GI com interfaces em 3D;

Realidade aumentada (RA): Nesta categoria foram incluídos os estudos em que os softwares de GI apresentam interfaces com realidade aumentada, como o uso de câmeras para capturar informações do mundo real e projetar virtualmente algum objeto;

Realidade virtual (RV): Nesta categoria foram incluídos os estudos em que os softwares de GI apresentam interfaces com realidade virtual, como o uso de óculos para recriar ao máximo a sensação de realidade ao indivíduo;

As categorias sendo como entrada de dados são as seguintes:

Teclado e mouse (TM): Os estudos incluídos nesta categoria apresentaram softwares em que os estudantes necessitam do teclado e mouse para interagir com a interface;

Baseada em caneta (BC): Esta categoria abrange interfaces que possuem como meio de interação por meio da caneta digital;

Baseada em toque (BT): Nesta categoria foram incluídos os estudos que exploram as interfaces com interação toque, abrangendo os diversos dispositivos que disponibilizam o multitoque e o toque único;

Háptica (HA): Esta categoria apresenta os artigos relacionados a interfaces táteis, sendo o principal objetivo para interfaces direcionadas aos deficientes visuais.

Dentre os 20 estudos que compõe a seleção final, dois estudos foram identificados como estudos que apresentam análise das interfaces de um modo geral. Esses estudos apresentam ideias abstratas ou boas práticas de desenvolvimento sem, no entanto, especificar nenhuma técnica, método, modelo ou abordagem de como estas interfaces foram desenvolvidas, e por essa razão nenhuma categoria foi criada para eles. 
A Figura 1 mostra a frequência dos estudos por categoria. Como mencionado anteriormente, alguns estudos foram agrupados em mais de uma categoria, afetando a sua frequência. A soma das frequências na Figura 1 (25) é maior do que o número de frequências na Tabela 2 (20).

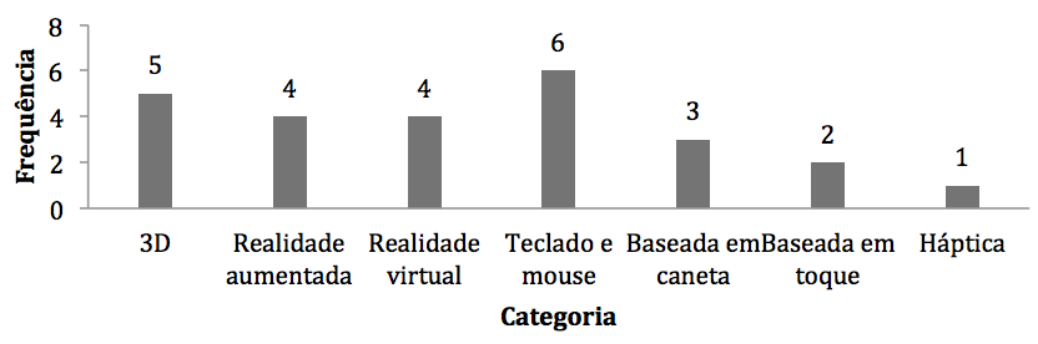

Figura 1 - Frequência de estudos em cada categoria

\section{Análise}

Esta seção apresenta uma visão geral de quais são as formas de interação (interfaces) entre os usuários e os softwares de GI identificadas. A partir da Figura 1, pode-se observar que a maioria dos estudos primários selecionados abordam o tipo de interação por teclado e mouse. Assim, a cargo desta informação, podemos concluir que a resposta à $\mathbf{Q P} \mathbf{P}_{1}$ é que há mais estudos das interfaces softwares de GI atuais por meio destes dois dispositivos. Em segundo lugar estão as interações por meio de 3D, com cinco estudos. As categorias "Realidade aumentada" e "Realidade virtual" apresentaram quatro estudos cada. Já a categoria "Baseada em caneta" apresentou três estudos. As categorias identificadas com menos estudos foram "Baseada em toque" e Háptica, com dois e um estudo respectivamente. É importante destacar que todos os estudos cuja interatividade ocorre por meio de interfaces em 3D, também apresentaram interação por meio da "Realidade aumentada", "Realidade virtual" ou "Teclado e mouse". Para verificar as tendências na área, a Figura 2 apresenta a relação entre as categorias dos estudos (eixo $\mathrm{x}$ ) e o ano de publicação (eixo y). A categoria "Realidade aumentada" apresentou dois estudos nos anos de 2006 e 2012. Porém, em 2013 apresentou somente um estudo.

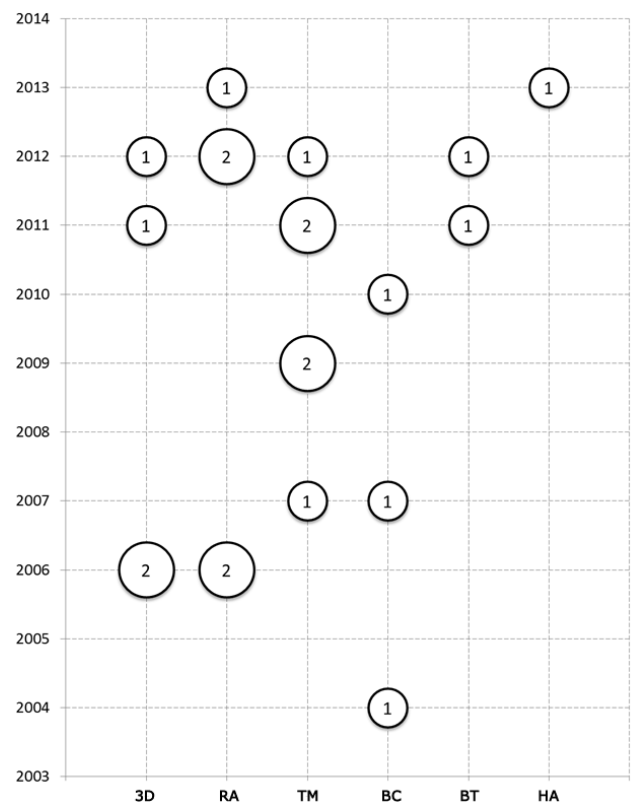

Figura 2 - Distribuição das categorias por ano 
A categoria "Teclado e mouse" apresentou um estudo primário em 2007, aumentando para dois estudos nos anos de 2009 e 2011 e depois somente um estudo em 2012. A categoria "Baseada em caneta" apresentou um estudo a cada três anos, sendo em 2004, 2007 e 2010. A categoria 3D apresentou dois estudos no ano de 2006, um estudo em 2012 e em 2011. As categorias como "Baseada em toque"(2011 e 2012) e Háptica (2013) apresentaram um estudo primário em cada ano.

Foram identificados também os diferentes tipos de dispositivos (devices) nos quais os softwares de GI são executados, o que permitiu responder a QP2. Foram encontrados sete tipos de dispositivos, sendo o computador de mesa o mais utilizado (12 estudos). Estudos com dispositivos móveis (e.g. PDA, Tablet, Tabletop), óculos e luvas para realidade virtual, e caneta digital foram utilizados em apenas um estudo cada. A Figura 3 apresenta todos os tipos de dispositivos que foram utilizados nos estudos primários.

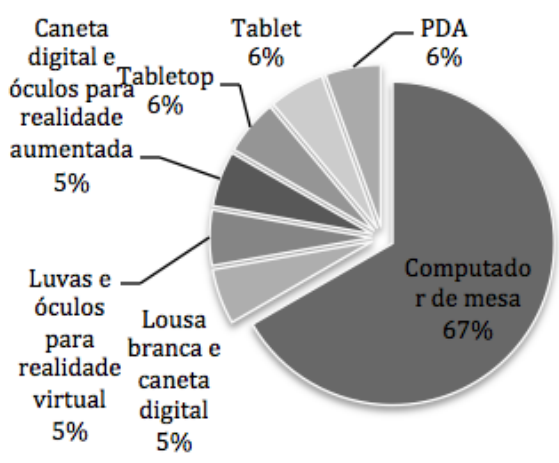

\begin{tabular}{l|c}
\hline Dispositivo & Qtde \\
\hline Computador de mesa & 12 \\
Lousa branca e caneta digital & 1 \\
Luvas e óculos para realidade virtual & 1 \\
Caneta digital e óculos para realidade aumentada & 1 \\
Tabletop & 1 \\
Tablet & 1 \\
PDA & 1 \\
\hline
\end{tabular}

Figura 3 - Distribuição dos estudos primários por tipo de publicação

Em relação aos tipos de publicação, foram analisados os estudos primários publicados em conferências, periódicos, simpósios e como capítulos de livros. A maioria dos estudos selecionados (nove estudos), foram publicados em conferências, em segundo lugar em periódicos, e em terceiro como capítulos de livro com dois estudos. Somente um estudo primário selecionado foi publicado em simpósio. A Figura 4 apresenta a quantidade de estudos primários de acordo com o tipo de publicação. É importante mencionar que as áreas de interesse dos estudos selecionados, em sua maioria, apresentam forte relação com diversas subáreas da ciência da computação e com educação.

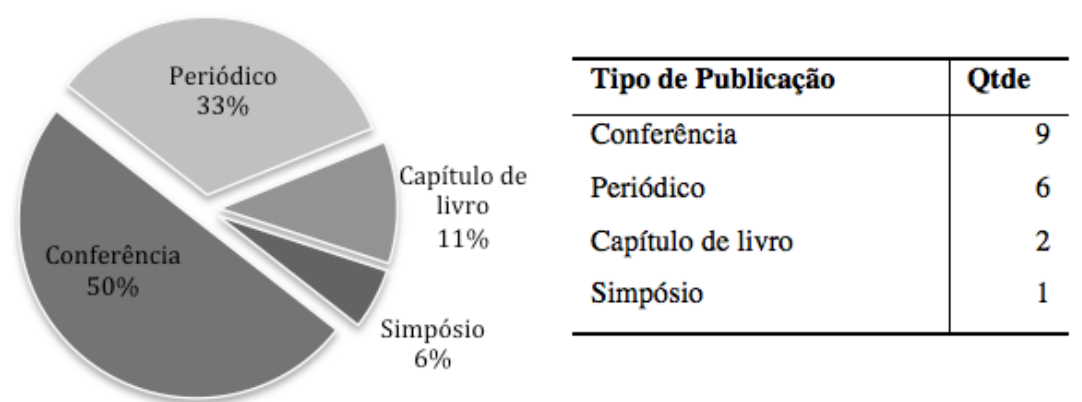

Figura 4 - Distribuição dos estudos primários pelo tipo da publicação

A Figura 5 apresenta um diagrama do tipo bubble chart que fornece uma visão mais precisa da frequência das ocorrências dos estudos primários selecionados de acordo com o tipo de contribuição e o tipo de pesquisa realizada de acordo com as 
definições propostas por Petersen (2008). O eixo y (centro) representa a categoria do estudo; à esquerda estão os tipos de contribuição, e à direita os tipos de pesquisa conduzida. $\mathrm{O}$ tamanho de cada bolha é determinada pelo número de estudos primários classificados como pertencentes aos pares correspondentes às coordenadas da bolha. Este resumo fornece uma visão panorâmica que possibilita identificar os estudos já realizados na área, juntamente com lacunas e oportunidades para futuras pesquisas.

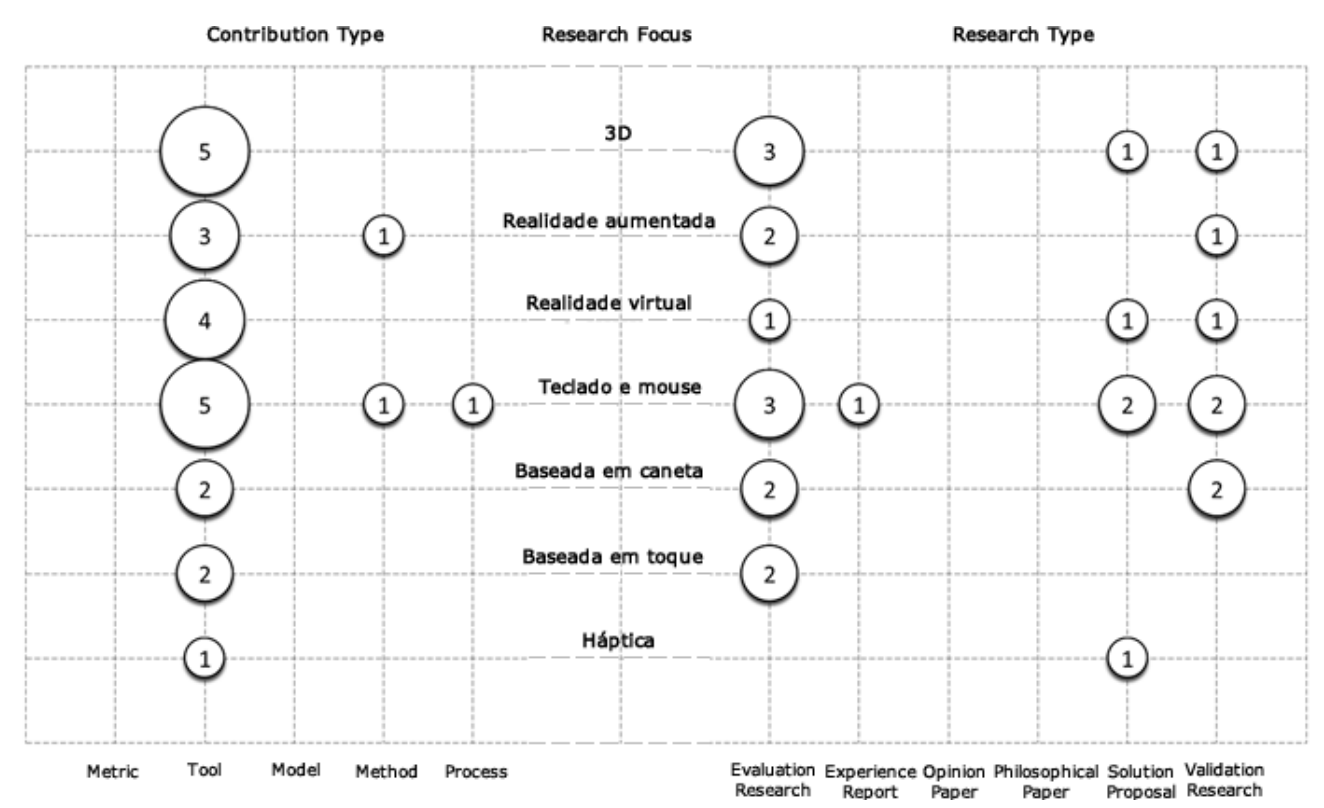

Figura 5 - Mapa da distribuição dos estudos por categoria

A tabela disponibilizada no endereço http://goo.gl/QzxGWn apresenta a lista dos estudos primários selecionados para este mapeamento, com os campos: autor, título do artigo, tipo de pesquisa, e tipo de contribuição.

\section{Síntese dos estudos do mapeamento}

Dez dos estudos encontrados fazem uso de teclado e mouse para prover interatividade ao software de GI. Dentre este dez, dois estudos investigam a relação entre o volume de informações apresentados nas interfaces, o consumo da carga cognitiva dos estudantes e seu impacto no aprendizado dos usuários (Reis et al. 2012; Schimpf e Spannagel 2012). Outro estudo com interação por meio do teclado e mouse é apresentado por Kovárová e Sokolský (2011), que analisaram os problemas encontrados nas interfaces dos softwares Cabri 3D e Archimedes Geo3D, e com os resultados desenvolveram um software com interface em 3D chamado Stereo3D. Este mesmo tipo de interação por teclado e mouse é discutido por Kortenkamp e Materlik (2004), na qual desenvolveram uma versão do software Cinderella para PDA. Neste caso, o mouse foi substituído por uma caneta digital.

No estudo apresentado por Bonnard et al. (2013) são utilizados um projetor e uma câmera para auxiliar na técnica de realidade aumentada. Alguns cartões com descrições de objetos geométricos são expostos em uma mesa. Cada um destes cartões contém um símbolo (markers) que é identificado por uma câmera e passa as informações recebidas para um software. Este software reconhece as descrições dos objetos que estão no cartão e realiza a projeção dos objetos em uma mesa, por meio de 
um projetor. Os desenhos dos objetos são atualizados ao mesmo tempo que estes cartões são manipulados dinamicamente.

Um estudo foi classificado em ambas categorias "Realidade aumentada" e "Realidade virtual". Neste estudo, Kaufmann e Schmalstieg (2006) desenvolveram um software de realidade virtual e aumentada, em que os objetos geométricos foram construídos em 3D e são visualizados por um óculos de realidade virtual. Estes objetos geométricos podem ser selecionados por meio de um menu que é projetado por realidade aumentada em um painel de vidro e manipulados por meio de uma caneta digital. Outro estudo selecionado na categoria "Realidade virtual" foi de Fabre et al. (2006). Neste estudo é apresentado um software com uma interface por meio da realidade virtual que permite a realização das atividades por meio da construção de objetos geométricos em 3D. A principal diferença entre o estudo de Kaufmann e Shmalstieg (2006) e Fabre et. al (2006) é que no primeiro estudo, a interação é por meio de uma caneta digital, diferentemente do segundo estudo, que é por meio de luvas.

Dois estudos foram classificados na categoria "Baseada em caneta". Tanto o estudo de Liu et al. (2007) quanto o de Song e Zhu (2010) utilizam uma caneta digital para reconhecer os objetos geométricos construídos por gestos utilizando a lousa branca. Porém, o estudo de Song e Zhu, ao invés da utilização da lousa branca, os alunos interagem por uma mesa digital substituindo o mouse, nos computadores de mesa. Em ambos softwares, é possível desenhar manualmente os objetos geométricos enquanto o sistema encarrega-se de reconhecer automaticamente o que está sendo desenhado.

Somente dois estudos selecionados foram categorizados como "Baseado em toque". No estudo de Blagojevic et al. (2012) são explorados o desenho de objetos geométricos por meio de ferramentas tradicionais como régua, compasso ou transferidor na superfície da tela do tablet. Apesar deste estudo não ser exclusivamente sobre softwares de GI, os autores enfatizam que esta ferramenta pode também ser utilizada para o ensino da geometria. Além deste estudo, Blanke e Schneider (2011) desenvolveram um software para Tabletop em que os alunos podem interagir com os objetos por meio de uma interface multitoque.

E finalmente, dois estudos não foram classificados em nenhuma categoria em especial,, apresentando metodologias de interação nas interfaces para estes softwares. Erez e Yerushalmy (2007) exploraram como a manipulação dos objetos geométricos pelo "arrasto do mouse" em tela pode afetar na percepção dos conceitos geométricos e no aprendizado da geometria. E no estudo de Mackrell (2011) apresenta uma análise como os componentes de uma tela devem ser desenvolvidos, como por exemplo a barra de ferramentas.

\section{Ameaças à Validade}

A fim de garantir uma seleção imparcial, as questões de pesquisa e os critérios de inclusão e exclusão foram definidos no início do mapeamento. Porém, não pode ser descartado a ameaça em relação a avaliação da qualidade dos estudos incluídos, em que foram selecionados sem qualquer atribuição de pontuação.

Outra possível ameaça identificada é a possibilidade de alguns artigos relevantes não serem incluídos, devido a utilização de um conjunto limitado de bibliotecas digitais. Além disso, o sistema de classificação e categorias elaborados representam também uma ameaça a validade. Como mostrado por Pretorius e Budgen (2008), a melhor 
maneira de classificar e categorizar os resultados só é obtido ao final da seleção. Além disso, o agrupamento dos estudos em mais de uma categoria pode representar uma ameaça potencial para contagem de frequência e as estatísticas deste estudo de mapeamento.

\section{Discussão e conclusão}

O mapeamento sistemático elaborado teve dois objetivos: primeiro, analisar quais tipos de interfaces (i.e. formas de interação) vem sendo desenvolvidas para os softwares de GI; e segundo, destacar em quais dispositivos estas interfaces são utilizadas. A maioria dos estudos encontrados preocupa-se em explorar a interação por meio do "Teclado e mouse", sendo que outros tipos de interação, como "Baseada em toque" ou Háptica são ainda pouco exploradas. Além disso, existem poucos softwares de GI desenvolvidos para outros dispositivos além dos computadores de mesa. Destaca-se ainda, que os resultados deste mapeamento apresentam indícios de que poucos estudos foram realizados focando no uso de técnicas e metodologias de Interação HumanoComputador para o desenvolvimento de interfaces para softwares de GI.

Além dos resultados obtidos, o mapeamento também aponta algumas oportunidades de pesquisa, tais como:

- Interface multitoque para os dispositivos com tela sensível ao toque: As interfaces multitoque nestes dispositivos podem proporcionar aos estudantes um aprendizado por meio de gestos naturais;

- Interface para pessoas com deficiência: O desenvolvimento de softwares de GI com interfaces especialmente desenvolvidas para dispositivos adequados aos portadores de necessidades especiais podem tornar os softwares de GI mais acessíveis a estes estudantes;

- Interfaces com luvas: Por meio de luvas os estudantes podem realizar a construção e o desenho de objetos geométricos pelo gesto;

- Interfaces com sensores de movimento: Os sensores de movimento podem ler os gestos dos estudantes e traduzir estes movimentos em comandos para o software de GI;

- Mesa digitalizadora: Por meio de uma mesa digitalizadora, os estudantes podem construir os objetos e manipulá-los com uma caneta digitalizadora, fazendo da mesma maneira que estivessem utilizando o lápis e o papel.

\section{Agradecimentos}

Os autores deste trabalho gostariam de agradecer o suporte financeiro proporcionado pela CAPES (Coodernação de Aperfeiçoamento de Pessoal de Nível Superior) e ao CNPq (Processo: 550449/2011-6 e 476566/2011-8).

\section{References}

Blagojevic, R., Chen, X., Tan, R., Sheehan, R., \& Plimmer, B. (2012). Using tangible drawing tools on a capacitive multi-touch display. Proceedings of the 26th Annual BCS Interaction Specialist Group Conference on People and Computers (pp. 315-320). Swinton, UK, UK: British Computer Society. 
Blanke, D., \& Schneider, G. (2011). TOM - A Multi-touch System for Learning Math. CSEDU (1)'11 (pp. 199-206).

Bonnard, Q., Jermann, P., Legge, A., Kaplan, F., \& Dillenbourg, P. (2012). Tangible paper interfaces: interpreting pupils' manipulations. Proceedings of the 2012 ACM international conference on Interactive tabletops and surfaces (pp. 133-142). New York, NY, USA: ACM.

Durelli H. S., Felizardo K. R., \& Delamaro M. D. (2010). Systematic mapping study on highlevel language virtual machines. In Virtual Machines and Intermediate Languages (VMIL '10). ACM, New York, NY, USA.

Erez, M. M., \& Yerushalmy, M. (2006). "If You Can Turn a Rectangle into a Square, You Can Turn a Square into a Rectangle..." Young Students Experience the Dragging Tool. International Journal of Computers for Mathematical Learning, 11(3), 271-299.

Fabre, A., Sternberger, L., Schreck, P., \& Bechmann, D. (2006). Constrained Gesture Interaction in 3D Geometric Constructions. In S. Gibet, N. Courty, \& J.-F. Kamp (Eds.), Gesture in Human-Computer Interaction and Simulation SE - 36 (Vol. 3881, pp. 324-334). Springer Berlin Heidelberg.

Isotani, S., \& Brandão, L. (2004). Ferramenta de avaliação automática no iGeom. Anais do Simpósio Brasileiro de Informática na Educação (SBIE) (pp. 328-337).

Kaufmann, H., \& Schmalstieg, D. (2006). Designing Immersive Virtual Reality for Geometry Education. Virtual Reality Conference, 2006 (pp. 51-58).

Kitchenham, B. Charters, S. Guidelines for performing systematic literature reviews in software engineering (version 2.3). Technical report, Keele University and University of Durham, 2007.

Kortenkamp, U., \& Materlik, D. (2004). Geometry teaching in wireless classroom environments using Java and J2ME. Science of Computer Programming, 53(1), 71-85.

Laborde, C. (2007). The role and uses of technologies in mathematics classrooms: Between challenge and modus vivendi. Canadian Journal of Science, Mathematics and Technology Education, 7(1), 68-92.

Mackrell, K. (2011). Design decisions in interactive geometry software. ZDM, 43(3), 373-387.

Petersen, K., Feldt, R., Mujtaba, S. \& Mattsson, M. (2008) Systematic mapping studies in software engineering. Proceedings of the 12th international conference on Evaluation and Assessment in Software Engineering, 68-77.

Pretorius, R. e Budgen, D. (2008) A Mapping Study on Empirical Evidence Related to the Models and Forms used in the UML. In 2nd ACM-IEEE International Symposium on Empirical Software Engineering and Measurement (ESEM), pages 342- 344. ACM.

Reis, H. M., Borges, S. S., Durelli, V. H. S., De S Moro, L. F., Brandao, A. A. F., Barbosa, E. F., Brandao, L. O., et al. (2012). Towards Reducing Cognitive Load and Enhancing Usability through a Reduced Graphical User Interface for a Dynamic Geometry System: An Experimental Study. Multimedia (ISM), 2012 IEEE International Symposium on (pp. 445450).

Roanes-Lozano, E. (2003). A bridge between dynamic geometry and computer algebra. Mathematical and Computer Modelling, 37(9-10), 1005-1028.

Schimpf, F., \& Spannagel, C. (2011). Reducing the graphical user interface of a dynamic geometry system. ZDM, 43(3), 389-397.

Shimomura, Y., Hvannberg, E., \& Hafsteinsson, H. (2013). Haptic cues as a utility to perceive and recognise geometry. Universal Access in the Information Society, 12(2), 125-142.

Song, X., \& Zhu, X. (2010). A Sketch Recognition Scheme for Primary Geometry Education. Biomedical Engineering and Computer Science (ICBECS), 2010 International Conference on (pp. 1-5). 